\title{
Mechanisms of hepatic encephalopathy and thiamine deficiency
}

\author{
Raghu Vemuganti • Alan S. Hazell
}

Published online: 29 June 2014

(C) Springer Science+Business Media New York 2014

Hepatic encephalopathy (HE) and thiamine deficiency (TD) represent two disorders in which metabolic derangements play a major role in their pathophysiology. They also define the areas of primary focus of Dr. Roger F. Butterworth, Ph. D., D.Sc. who retired from the University of Montreal in the summer of 2013. This special issue of Metabolic Brain Disease commemorates his valuable contribution to the study of these maladies.

Although HE is considered a metabolic disorder, new evidence is emerging to show that it has a molecular basis as well. Vemuganti et al. reports altered cerebral profiles of microRNAs after acute liver failure (ALF) that likely contribute in a significant way to gene expression changes associated with the ensuing HE. Disrupted ammonia detoxification is considered a major precipitator of neurological dysfunction in HE, and current understanding of the contribution of ammonia in liver diseases is discussed in an article by Ott and Vilstrup, while the effects of hyperammonemia on cerebral metabolic function are focussed on in an article by Schousboe and colleagues. In addition, impaired brain energy metabolism is a serious complicating feature of liver failure, with lactate production being a major consequence of this problem. In relation to this, the role of increased lactate accumulation and its pathogenetic basis in HE is discussed by Bosoi and Rose. Also, a major and often lethal complication of ALF is brain edema, and the significance of this edema and its underlying basis in ALF-induced HE is examined by Rama Rao

R. Vemuganti $(\bowtie)$

Department of Neurological Surgery, University of Wisconsin, Madison, WI, USA

e-mail: vemuganti@neurosurgery.wisc.edu

A. S. Hazell $(\bowtie)$

Department of Medicine, University of Montreal, Montreal, QC, Canada

e-mail: alan.stewart.hazell@umontreal.ca et al., while the usefulness of magnetic resonance imaging as a tool in both acute and chronic liver failure is considered by Chavarria and Cordoba.

Minimal hepatic encephalopathy (MHE) represents the mildest of the spectrum of HE forms in which patients do not display clinical symptoms but have mild cognitive and psychomotor deficits. It is also associated with poor survival rates. Two papers examine aspects of MHE, in which cognitive impairment and the issue of cognitive variability as a contributing factor in these patients is a focus of attention in Bisiacchi et al., and in an experimental model of chronic liver failure in which focal increases in the binding of translocator $(18 \mathrm{kD})$ protein in brain is reported by Agusti et al. Increasingly, the phenomenon of acute-on-chronic liver failure, in which acute deterioration of liver function in patients with cirrhosis is a consequence, is being considered a distinct clinical entity. The subject is discussed in Wright et al. In addition, considerable evidence has shown that astrocyte dysfunction plays a major role in HE. Karabara and colleagues explore the potential role of GlcNAcylation in these cells, while the protective effect of inhibition of glutamine synthesis is examined in neurological diseases, including HE, by Jeitner and Cooper.

Development of well-defined biomarkers and improved therapeutic targets represents an important subject regarding HE. In this regard, the paper by Cooper and Kuhara shows that $\alpha$-ketoglutaramate is a good marker of HE, and the paper by Mondal and Trigun demonstrates that pannexin-1 can be a marker as well as a target for HE. Although the mechanism (s) underlying the beneficial effects of rifaximin in HE remain largely unknown, the topic is tackled using multi-modal MRI in Ahluwalia et al. In addition, the subject of microglial proliferation and its significance in alcoholics with HE is explored experimentally in Dennis et al. Dienel and Cruz address the subject of decreased proteolysis as a result of hyperammonemia due to liver failure, and the effects of 
thioacetamide-induced ALF on citrulline uptake is examined in a study by Zielińska et al.

Finally, Roger's interest in the pathophysiology of TD has been long and enduring. A number of papers in this special issue deal with different aspects of TD. Afadlal et al. shows that astrocytes play a significant role in TD-induced encephalopathy, while Bettendorff explores the role of thiamine triphosphate in cells. In addition, since mitochondrial dysfunction is an important aspect of both TD and Alzheimer's disease in which thiamine-dependent enzyme activity is reduced, Huang et al. explores how cellular calcium stores are affected in a fibroblast cell line, an important first step towards the eventual determination of how this process comes about in the $\mathrm{AD}$ patient and development of protective/treatment strategies targeting this mechanism, which may also have relevance for the future treatment of TD.

ASH: I first met Roger Butterworth in 1991 as a doctoral student in Tony Hakim's group at the Montreal Neurological Institute, McGill University. At the time we were looking to measure amino acid levels, particularly glutamate, in brain extracellular fluid in TD, the underlying basis of Wernicke's encephalopathy, and Roger's Neuroscience Research Unit at the University of Montreal which regularly performed amino acid measurements in brain tissue seemed an appropriate place to start our enquiries. I was immediately struck by Roger's relaxed, easy going personality. An important collaboration was quickly established between the two groups which eventually led to the first direct evidence for the existence of an excitotoxic event in vulnerable brain regions in TD. In 19962000 I was fortunate enough to be able to work in Roger's laboratory as a post-doctoral fellow; his valuable mentorship has helped define significantly my approach to research. Following my subsequent recruitment at the University of Montreal in 2000 Roger was a major source of inspiration; I will always value our often lengthy research discussions in and around our offices.

$\mathrm{RV}$ : While working for my $\mathrm{PhD}$ thesis on glutamate neurotransmission in hyperammonemia, I read many seminal papers by Roger's group. Surprisingly, in 1991 when I submitted my thesis, my University requested Roger to serve as the external examiner. My thesis exam was the best example of Roger's magnanimity as he ignored all my mistakes and gave credit for the data. I subsequently joined Roger's lab as a post-doc in January 1992. Roger received me at the Mirabel airport which shows his kind heart. My research blossomed at Roger's lab. Roger's method of working with post-docs and graduate students was amazing. He respected our scientific ideas, allowed us to plan our projects, always had time to discuss the research and his critiques were unbiased. I wish I could be as compassionate and scientifically mature as Roger when dealing with my own students. He gave me enormous confidence and freedom to freely experiment, at the same time gently nudged me whenever I weaned from the path. I have met very few people who are as generous as Roger. I have no words to express my gratitude towards him for giving me the first break in my life. I wish him all the best in retirement.

ASH and RV: Altogether, this special issue contains articles that are examples of our current understanding of $\mathrm{HE}$ and TD, providing insight into their complexity. Roger, we thank you for all your years of dedication and commitment to these two areas of research. Most of all, thank you for helping to shape our careers in a major way. 